of the neuropathy. After three months the diplopia had disappeared. The CSF showed $6 \times 10^{9} / 1$ mononuclear cells and a protein content of $0.88 \mathrm{~g} / 1$; some oligoclonal bands were present, but the IgG index was normal. Brain MRI showed multiple abnormalities of periventricular white matter. Some of these enhanced after intravenous administration of gadolineum, suggesting active CNS inflammation. In the pons an area of high T2 signal intensity was seen in the proximal part of the fila radicularia of the left abducens nerve, providing substantial evidence for a sixth nerve palsy of CNS origin (figure).

Although a vascular origin cannot be fully excluded, the distribution of the lesions was typical for plaques seen in multiple sclerosis. Moreover, the enhancing white matte lesions were present several weeks after onset of the sixth nerve palsy, making a coincidental vascular lesion unlikely. Lesions without enhancement may reflect older lesions. Nevertheless, multiple sclerosis could not be diagnosed, as other white matter abnormalities in the CNS were asymptomatic.

Previous studies have suggested that cranial nerve lesions in CIDP may be related to CNS lesions. ${ }^{15}$ However, MRI abnormalities were not more common in patients with cranial nerve involvement than in those without. ${ }^{1}$ The enhancing pontine lesion in our patient is suggestive of active CNS demyelination and therefore probably responsible for a central origin of the ocular palsy, which interestingly occurred independent of peripheral nerve involvement at that time. Previous information on white matter abnormalities in the CNS in this patient was not obtained as MRI is not a routine diagnostic procedure in CIDP. Yet these abnormalities may occur in a disproportionate number of patients. ${ }^{1367}$

There may be several explanations for the combined occurrence of central and peripheral myelin dysfunction. Firstly, coincidence seems unlikely, as so many patients with CIDP show evidence of involvement of central white matter. Secondly, the process of demyelination in itself may lead to ongoing generalised demyelination through activation of cytokines. Finally, central and

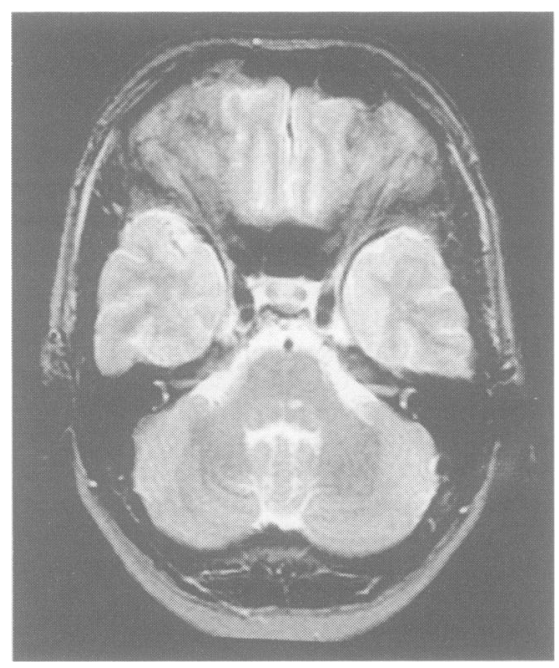

$T 2$ weighted transaxial spin echo $M R$ study (TR 2653, TE 100). At the level of the middle cerebellar peduncles an isolated lesion of high signal intensity is present below the floor of the fourth ventricle, close to the median plane, corresponding with the proximal part of the fila radicularia of the left abducens nerve. peripheral myelin may share common antigens which trigger an immune response. The occurrence of isolated sixth nerve palsy in our patient stresses the relevance of MR in patients with CIDP and cranial nerve dysfunction.

We thank Dr L Jaap Kappelle for his comments.

JOHN HJ WOKKE

LEONARD H VAN DEN BERG Department of Neurology JAN PJ VAN SCHAIK

The Rudolf Magnus School in the Neurosciences, University Hospital Utrecht, University Hospital Utrecht,
PO Box 85500, 3508 GA Utrecht The Netherlands

Correspondence to: Dr Wokke.

1 Ormerod IEC, Waddy HM, Kermode AG Murray NMF, Thomas PK. Involvement of the central nervous system in chronic inflammatory demyelinating polyneuropathy: a clinical, electrophysiological and magnetic resonance study. $f$ Neurol Neurosurg Psychiatry 1990;53:789-93.

2 Thomas PK, Walker RWH, Rudge P, et al. Chronic demyelinating peripheral neuropathy associated with multifocal central nervous system demyelination. Brain 1987; vous system

3 Donaghy M, Earl CJ. Ocular palsy preceding chronic relapsing polyneuropathy by several chronic relapsing polyneuropathy

4 Van den Berg LH, Mollee I, Wokke JHJ, Logtenberg $T$. Increased frequencies of HPRT mutant $T$ lymphocytes in patients with Guillain-Barré syndrome and chronic inflammatory demyelinating polyneuropathy further evidence for a role of $T$ cells in etiopathogenesis of peripheral demyelinatin diseases. $\Im$ Neuroimmunol 1995;58:37-42.

5 Waddy HM, Misra VP, King RHM, et al. Focal cranial nerve involvement in CIDP. clinical and MRI evidence of peripheral and clinical and $\mathrm{nRT}$ evidence of peripheral and $400-5$.

6 Rubin M, Karpati G, Carpenter S. Combined central and peripheral myelinopathy. central and peripheral
Neurology 1987;37:1287-90.

7 Feasby TE, Hahn AF, Koopman WJ, Lee DH. Central lesions in chronic inflammatory demyelinating polyneuropathy. Neurology 1990;40:476-8.

\section{Capture-recapture methods for precise measurement of the incidence and prevalence of stroke}

Capture-recapture methods, originally developed for wildlife censuses, are increasingly being used in epidemiology to estimate the incidence and prevalence of disease in the population. ${ }^{1}$ The principle is that two or more censuses are taken, with the people or animals being identified and marked on each occasion, and knowledge of the degree of independence of these censuses is used to estimate the proportion of the total population that has been missed. This allows an estimate of the size of the total population under study. Capture-recapture results have been published for a wide range of medical conditions including myocardial infarction, ${ }^{2}$ but not yet for stroke. The method is attractive because maintaining community based registers that are "as complete as possible" is an expensive exercise.

Using the capture-recapture method, at least two independent sources of cases need to be identified. The number of cases that are common to both sources is calculated $(\mathrm{m})$, as are also the total numbers of cases from the first and second samples ( $M$ and $n$ respectively). A simple capture-recapture estimate of the total number of cases, $N$, is given by the equation:

$$
N=\frac{(M+1)(n+1)}{(m+1)}-1
$$

LaPorte has used the capture-recapture method to estimate the number of cases of myocardial infarction in six communities in Sweden, ${ }^{2}$ with the myocardial infarction registry as the first source $(M=5832)$ and the hospital discharge index as the second source ( $n=6582)$, and noted an overlap of $m=4746$. The capture-recapture estimate of 8088 cases implies that $5 \%$ of the cases of myocardial infarction had been missed by both sources of notification. The fact that cases may be missing from the individual sources need not be an important drawback when the capture-recapture method is used, as the pattern of overlap allows these missing cases to be inferred.

In a preliminary analysis we have attempted to apply capture-recapture to a previously published study of the incidence of first time stroke among those aged under 75 years. $^{3}$ This was carried out in three districts of south east England (two inner city and one rural) in 1989-91 and used notifications from hospital ward registers, general practitioners, rehabilitation staff, and death certificates. The main difficulty we met was the lack of independence between sources of notification, which would be due partly to strokes of different degrees of severity being likely to be registered by the different sources. (This is known as "variable catchability" from the corresponding situation in wildlife surveys, in which some categories of animal are more likely to be caught in one census than in another.) In our study, an example of variable catchability is that the most serious strokes would most likely be registered only as death certificates, and the mildest strokes only as general practitioner or rehabilitation staff notifications.

Secondly, when planning a study in which capture-recapture methods are not intended, it is natural to encourage sources of notification to be complementary rather than overlapping. For instance, in our study general practitioners were asked to record all cases of stroke but to take particular care to record those not admitted to hospital.

A further difficulty, especially in inner city areas, is the high mobility of the overall population - that is, those people resident in the study districts and at risk of stroke. The overall annual population mobility in central London is estimated at around $30 \%$, but this would be lower for the older people more likely to have a stroke.

Log linear modelling is now the most common statistical method for analysing capture-recapture data. It is flexible but does not allow for variable catchability, therefore the analysis should be stratified-that is, the different types of case (for example, mild, moderate, and severe) should be identified and separate estimates made of their respective sizes. Calculating confidence intervals for incidence estimates is not straightforward, but the "bootstrapping" computational technique has been used.

In our incidence study a total of 639 strokes were registered, representing a crude annual rate of 0.68 patients with first time stroke per 1000 population (aged under 75 years). ${ }^{3}$ The corresponding crude rate from the Oxfordshire Community Stroke Project, some 10 years earlier, was 0.93 with $95 \%$ confidence interval $0 \cdot 82-1 \cdot 04 .^{5}$

Our initial capture-recapture estimate of the total number of strokes, obtained using $\log$ linear modelling, was 1999 , corresponding to $2 \cdot 13$ cases per 1000 person-years. This rate is more than twice that of the Oxfordshire study and, considering the thor- 
ough case collection methods of our study, ${ }^{3}$ implies an inconceivably low ascertainment rate for our study of only $32 \%$. It would be reasonable to expect the pattern of notification (by hospital ward registers, general practitioners, and rehabilitation staff) to differ between patients who died and those who did not; separate capture-recapture estimates were therefore calculated for these two groups to obtain a stratified estimate, but their total was similar to the estimate previously obtained.

- If capture-recapture techniques are to be useful for estimating the incidence of stroke, then this should be incorporated into the design of the study

- When using capture-recapture, underascertainment is not itself a problem but it is vital that multiple notification of patients is maintained
- Patients should be stratified according to different relative probabilities of being notified by the sources. Strata should be defined in terms of some objective characteristic, rather than by survey results (as we used in the above example)

- Capture-recapture methods have great potential for increasing the efficiency of surveys to estimate the incidence and prevalence of stroke.

At least until we have more experience in the application of capture-recapture, we must continue to attempt complete coverage of cases with stroke registers.

N A TAUB C-STO WOLFE Department of Public Health Medicine, UMDS, St Thomas's Campus, London SE1 7EH, UK

Correspondence to: N A Taub, Department of
Epidemiology and Public Health, 22-28 Princess Road West, Leicester LE1 6TP, UK

1 LaPorte RE. Assessing the human condition: capture-recapture techniques. $B M \mathcal{F} 1994$ 308:5-6.

2 LaPorte RE, Tull ES, McCarty D. Monitoring the incidence of myocardial infarctions: applications of capture-recapture technology Int $f$ Epidemiol 1992;21:258-63.

3 Wolfe CDA, Taub NA, Woodrow J, Richardson E, Warburton FG, Burney PGJ Does the incidence, severity or case fatality of stroke vary in southern England? Epidemiol Community Health 1993;47: $139-43$.

4 Smith EP, van Belle G. Nonparametric estimation of species richness. Biometrics 1984;40 119-29.

5 Malmgren R, Bamford J, Warlow C, Sandercock P, Slattery J. Projecting the number of patients with first ever strokes and number of patients with first ever strokes and patients newly handicapped by stroke in
England and Wales. BMF 1989;298:656-60.
Clinical and genetic evaluation of Japanese autosomal dominant cerebellar ataxias; is Machado-Joseph disease common in the Japanese?

Direct mutation analysis for MachadoJoseph disease, ${ }^{1}$ dentatorubral-pallidoluysian atrophy, ${ }^{2}$ and spinocerebellar ataxia type $1,{ }^{3}$ with which CAG repeat expansions are associated, allows the molecular diagnosis of autosomal dominant cerebellar ataxia (ADCA). Due to the variable clinical manifestations and age of onset in each patient with ADCA, which are correlated with the triplet repeat length, ${ }^{1-3}$ it is often difficult to diagnose patients with ADCA from clinical investigations alone, especially distinguishing between MachadoJoseph disease and spinocerebellar ataxia type $1 .^{4}$ In Japan, Machado-Joseph disease is considered uncommon and Menzel type of hereditary cerebellar ataxia, which is a clinical entity corresponding to ADCA type I except for Machado-Joseph disease, ${ }^{5}$ is often diagnosed, presumably, even in patients with Machado-Joseph disease. Thus the accuracy of the clinical classification of ADCA may be questioned.

We examined a series of 32 patients from 29 Japanese ADCA families, from a region having a population of seven million, to confirm their genetic diagnosis by PCR amplification and re-evaluated their clinical characteristics from the viewpoint of the genotype-phenotype correlation (table). Genomic DNA was extracted from blood cells and each disease locus containing triplet repeats was amplified by PCR. The primers and conditions of the reaction for dentatorubral-pallidoluysian atrophy and spinocerebellar ataxia type 1 have been described. ${ }^{23}$ For Machado-Joseph disease, we originally designed primers MJ1 (5'TGATTCGTGAAACAATGTATT-3') and MJ2 (5'-AGGTAGCGAACATGATGAATG-3'). Products of PCR were electrophoresed on $2 \%$ agarose gel followed by ethidium bromide staining or $6 \%$ denaturing acrylamide gel with silver stain plus kit (Bio $\mathrm{Rad})$. The repeat length of the expanded alleles was calculated in comparison with the sequenced sample using a video densitometer (Bio-profil system (Vilber Lourmat)).

Eighteen patients from 17 families, constituting $56 \%$ of all patients with ADCA, had an abnormal expansion in the Machado-Joseph disease locus with various repeat lengths. The age of onset was highly correlated with the number of repeats $(r=$ $-0.79 ; \mathrm{P}<0.001$ ), as reported elsewhere. All patients with Machado-Joseph disease presented with nystagmus and cerebellar ataxia. They also had hyperactive limb reflexes, ophthalmoplegia, and spasticity. Dystonia was prominent in the patients with a longer repeat number and earlier onset. However, amyotrophy and sensory disturbance appear in patients with late onset or severely advanced disease. No involuntary movement was identified. Most of these manifestations were also found in patients with spinocerebellar ataxia type 1 .

Summary of the clinical and genetic data on fapanese ADCA

\begin{tabular}{|c|c|c|c|c|c|c|c|c|c|c|c|c|c|c|c|c|}
\hline Case & Age/sex & Onset & Ataxia & $\begin{array}{l}\text { Deep } \\
\text { tendon } \\
\text { reflexes }\end{array}$ & $\begin{array}{l}\text { Ophthal- } \\
\text { moplegia }\end{array}$ & $\begin{array}{l}\text { Nystag- } \\
\text { mus }\end{array}$ & $\begin{array}{l}\text { Involun- } \\
\text { tary } \\
\text { movement }\end{array}$ & Dystonia & $\begin{array}{l}\text { Amy- } \\
\text { otrophy }\end{array}$ & $\begin{array}{l}\text { Sensory } \\
\text { disturb- } \\
\text { ance }\end{array}$ & $\begin{array}{l}\text { Spas- } \\
\text { ticity }\end{array}$ & $\begin{array}{l}\text { Epi- } \\
\text { lepsy }\end{array}$ & $\begin{array}{l}\text { Demen- } \\
\text { tia }\end{array}$ & $\begin{array}{l}\text { Clinical } \\
\text { diag- } \\
\text { nosis }\end{array}$ & $\begin{array}{l}\text { Mole- } \\
\text { cular } \\
\text { diagnosis }\end{array}$ & $\begin{array}{l}\text { No of } \\
\text { CAG } \\
\text { repeats * }\end{array}$ \\
\hline 1 & $67 \mathrm{~F}$ & 50 & + & $\uparrow$ & + & + & - & + & + & + & + & - & - & Menzel & MJD & 69 \\
\hline 2 & $47 \mathrm{M}$ & 30 & + & $\uparrow$ & + & + & - & + & + & + & + & - & - & Menzel & MJD & 73 \\
\hline 3 & $66 \mathrm{M}$ & 60 & + & $\rightarrow$ & + & + & - & - & - & + & - & - & - & MJD & MJD & 64 \\
\hline 4 & $52 \mathrm{~F}$ & 46 & + & $\uparrow$ & + & + & - & - & - & + & + & - & - & MJD & MJD & 69 \\
\hline 5 & $48 \mathrm{~F}$ & 35 & + & $\uparrow$ & + & + & - & + & - & - & - & - & - & Menzel & MJD & 66 \\
\hline 6 & $61 \mathrm{M}$ & 49 & + & $\uparrow$ & + & + & - & - & + & + & + & - & - & Menzel & MJD & 65 \\
\hline 7 & $51 \mathrm{~F}$ & 34 & + & $\uparrow$ & + & + & - & + & - & + & + & - & - & MJD & MJD & 67 \\
\hline 8 & $38 \mathrm{M}$ & 32 & + & $\uparrow$ & + & + & - & - & - & - & - & - & - & MJD & MJD & 69 \\
\hline 9 & $42 \mathrm{M}$ & 24 & + & $\uparrow$ & + & + & - & - & - & - & + & - & - & MJD & MJD & 74 \\
\hline 10 & $46 \mathrm{~F}$ & 36 & + & $\uparrow$ & + & + & - & + & - & - & + & - & - & MJD & MJD & 65 \\
\hline 11 & $51 \mathrm{~F}$ & 41 & + & $\uparrow$ & + & + & - & - & + & + & + & - & - & MJD & MJD & 67 \\
\hline 12 & $51 \mathrm{~F}$ & 33 & + & $\uparrow$ & + & + & - & + & + & + & + & - & - & MJD & MJD & 73 \\
\hline 13 & $53 \mathrm{~F}$ & 49 & + & $\uparrow$ & + & + & - & - & - & - & + & - & - & MJD & MJD & 64 \\
\hline 14 & $71 \mathrm{M}$ & 52 & + & $\uparrow$ & + & + & - & - & + & + & + & - & - & Menzel & MJD & 64 \\
\hline 15 & $60 \mathrm{M}$ & 50 & + & $\rightarrow$ & + & + & - & - & - & - & + & - & - & MJD & MJD & 66 \\
\hline 16 & $48 \mathrm{~F}$ & 34 & + & $\uparrow$ & + & + & - & - & + & - & + & - & - & MJD & MJD & 68 \\
\hline 17 & $37 \mathrm{~F}$ & 25 & + & $\uparrow$ & + & + & - & + & + & + & + & - & - & MJD & MJD & 73 \\
\hline 18 & $48 \mathrm{~F}$ & 40 & + & $\uparrow$ & + & + & - & - & - & - & + & - & - & MJD & MJD & 68 \\
\hline 19 & $53 \mathrm{~F}$ & 41 & + & $\uparrow$ & - & - & - & - & - & - & + & - & - & Menzel & SCAl & \\
\hline 20 & $28 \mathrm{M}$ & 17 & + & $\rightarrow$ & + & - & - & - & + & - & + & - & - & Menzel & SCAl & \\
\hline 21 & $61 \mathrm{~F}$ & 46 & + & $\uparrow$ & - & - & - & - & - & - & - & - & + & Menzel & SCA1 & \\
\hline 22 & $47 \mathrm{M}$ & 43 & + & $\rightarrow$ & - & + & - & - & + & - & - & - & - & ADCAIII & ND & \\
\hline 23 & $71 \mathrm{~F}$ & 49 & + & $\rightarrow$ & - & + & - & - & + & - & - & - & - & ADCAIII & ND & \\
\hline 24 & $27 \mathrm{M}$ & 20 & + & $\rightarrow$ & - & + & - & - & + & - & - & - & - & ADCAIII & ND & \\
\hline 25 & $39 \mathrm{M}$ & 30 & + & $\downarrow$ & - & SEM & - & - & - & - & - & - & - & Menzel & ND & \\
\hline 26 & $35 \mathrm{M}$ & 20 & + & $\downarrow$ & + & SEM & + & - & - & - & - & - & - & Menzel & ND & \\
\hline 27 & $38 \mathrm{~F}$ & 32 & + & $\downarrow$ & - & SEM & + & - & - & - & - & - & - & Menzel & ND & \\
\hline 28 & $55 \mathrm{~F}$ & 50 & + & $\rightarrow$ & - & - & + & - & - & - & - & + & + & DRPLA & DRPLA & \\
\hline 29 & $46 \mathrm{~F}$ & 38 & + & $\rightarrow$ & - & - & + & - & - & - & - & + & + & DRPLA & DRPLA & \\
\hline 30 & $30 M$ & 19 & + & $\rightarrow$ & - & - & + & - & - & - & - & + & + & DRPLA & DRPLA & \\
\hline 31 & $28 \mathrm{M}$ & 12 & + & $\rightarrow$ & - & - & - & + & - & - & - & + & + & DRPLA & DRPLA & \\
\hline 32 & $29 \mathrm{M}$ & 14 & + & $\rightarrow$ & - & - & - & - & - & - & - & + & + & DRPLA & DRPLA & \\
\hline
\end{tabular}

*All samples were heterogenous for the mutations and only expanded alleles were counted.

$\mathrm{ND}=$ not determined, cases 25 to 27 were clinically suspected as having spirocerebellar ataxia type 2 ; SEM = slow eye movement without nystagmus; Menzel = Menzel type of hereditary cerebellar ataxia; DRPLA = dentatorubral-pallidoluysian atrophy. 